\title{
Identification of the Boudicca and Sinbad retrotransposons in the genome of the human blood fluke Schistosoma haematobium
}

\author{
Claudia S Copeland/*/+ , Fred A Lewis**, Paul J Brindley
}

\begin{abstract}
Department of Tropical Medicine, Tulane University Health Sciences Center, New Orleans, LA, US *United States Department of Agriculture, Associated Research Service, Center for Medical, Agricultural, and Veterinary Entomology, Gainesville, FL, US

**Biomedical Research Institute, Rockville, MD, US
\end{abstract}

Schistosomes have a comparatively large genome, estimated for Schistosoma mansoni to be about 270 megabase pairs (haploid genome). Recent findings have shown that mobile genetic elements constitute significant proportions of the genomes of S. mansoni and S. japonicum. Much less information is available on the genome of the third major human schistosome, S. haematobium. In order to investigate the possible evolutionary origins of the S. mansoni long terminal repeat retrotransposons Boudicca and Sinbad, several genomes were searched by Southern blot for the presence of these retrotransposons. These included three species of schistosomes, S. mansoni, S. japonicum, and S. haematobium, and three related platyhelminth genomes, the liver flukes Fasciola hepatica and Fascioloides magna and the planarian, Dugesia dorotocephala. In addition, Homo sapiens and three snail host genomes, Biomphalaria glabrata, Oncomelania hupensis, and Bulinus truncatus, were examined for possible indications of a horizontal origin for these retrotransposons. Southern hybridization analysis indicated that both Boudicca and Sinbad were present in the genome of S. haematobium. Furthermore, low stringency Southern hybridization analyses suggested that a Boudicca-like retrotransposon was present in the genome of $\mathrm{B}$. truncatus, the snail host of $\mathrm{S}$. haematobium.

Key words: schistosome - long terminal report retrotransposon - mobile genetic element - reverse transcriptase - Bulinus

The life cycle of schistosomes, diecious trematodes of the order Digenea, involves parasitism of both humans and genera of aquatic or amphibious snails specific to each species: Biomphalaria for Schistosoma mansoni, Oncomelania for S. japonicum, and Bulinus for S. haematobium. In the case of $S$. mansoni and $S$. japonicum, adult worms inhabit the portal system blood vessels and mesenteric veins of the intestines of their human hosts. Much progress has been made toward sequencing and sequence annotation of the genomes of $S$. mansoni and S. japonicum (El-Sayed et al. 2004), including characterization of much of their transcriptomes (Hu et al. 2003, Verjovski-Almeida et al. 2003), and the construction of a bacterial artificial chromosome (BAC) library representing an eight-fold coverage of the $S$. mansoni genome (Le Paslier et al. 2000). In contrast to the burgeoning wealth of sequence information for S. mansoni and S. japonicum, relatively little is known about the genome sequence of $S$. haematobium; less than seventy S. haematobium nucleotide sequences were present in GenBank as of February 2006, compared with over 100,000 each for $S$. mansoni and S. japonicum. The genomic DNA of Schistosoma is indistinguishable among S. mansoni, S. japonicum, and

Financial support: Ellison Medical Foundation (Infrastructure grant award ID-IA-0037-02 to PJB), NIAID-NIH (supply contract NO1-A1-55270 to FL). PJB is a recipient of a Burroughs Wellcome Fund Scholar Award in Molecular Parasitology.

${ }^{+}$Corresponding author: ccopelan @ gainesville.usda.ufl.edu Received 23 February 2006

Accepted 8 June 2006
S. haematobium by physical measurement (Hilyer 1974, Rollinson et al. 1997) and their chromosomal karyotypes are similar in appearance (Hirai et al. 2000).

Mobile genetic elements (MGEs) represent a major force driving the evolution of eukaryotic genomes (Charlesworth et al. 1994, Kidwell \& Lisch 1997, Kazazian 2004) and play an important role in the establishment of genome size (Petrov et al. 2000). In schistosomes, more than half of the genome appears to be composed of, or derived from, repetitive sequences, to a large extent from retrotransposable elements (Brindley 2005, Copeland et al. 2005b). Schistosome MGEs include SINE-like retroposons (Spotila et al. 1989, Drew \& Brindley 1995), long terminal repeat (LTR) retrotransposons (Laha et al. 2001, 2004, Foulk et al. 2002, Copeland et al. 2003, 2005a, DeMarco et al. 2004), non-LTR retrotransposons (Laha et al. 2002a, b, Brindley et al. 2003), and DNA transposons related to bacterial IS1016 insertion sequences (Feschotte 2004). The long terminal repeat retrotransposable elements, i.e. the LTR retrotransposons, are similar in structure and life cycle to retroviruses (Garfinkel et al. 1991, Havecker et al. 2004), differentiated from true retroviruses mainly by their lack of an envelope-encoding gene. They are of interest for their potential for horizontal transmission, as well as their ability to shed light on phylogenies of their host organisms when solely vertically transmitted.

Boudicca, the first fully characterized LTR retrotransposon from the genome of $S$. mansoni (Copeland et al. 2003, 2004), belongs to the gypsy-like retrotransposons, one of three distinct major groups of LTR retrotransposons: the Gypsy/Ty3 group, the Copia/Tyl group and the Pao/BEL group (Xiong et al. 1993). Sinbad, another LTR retrotransposon from $S$. mansoni, is a member 
of the Pao/BEL group (Copeland et al. 2005a). Here, we investigated the possibility of the presence of homologues of Boudicca and Sinbad in genomes phylogenetically related to $S$. mansoni and genomes of host species parasitized by these schistosomes, using Southern hybridization analyses. Six platyhelminths were examined including the three major schistosome species, S. mansoni, $S$. japonicum, and $S$. haematobium, two non-schistosome trematodes, Fasciola hepatica and Fascioloides magna, and a (free-living) planarian, Dugesia dorotocephala. D. dorotocephala, a member of the class Turbellaria, served as a representative of platyhelminths phylogenetically distant from the schistosomes. The liver flukes $F$. hepatica and $F$. magna, like schistosomes, are members of class Trematoda, order Digenea. Unlike the schistosomes, flukes of this family are monecious, live as adults within the ducts of the liver rather than the blood vessels, and enter the host through the ingestion of vegetation contaminated with encysted larval forms known as metacercariae. F. hepatica, the sheep liver fluke, is primarily a parasite of sheep and other domesticated ruminants, but is also known to infect humans. F. magna, the deer liver fluke (renowned for its remarkable size and also known as the "giant fluke" and the "large American liver fluke") is a wildlife parasite of deer, but can also infect other large ruminants, including cattle and sheep (Swales 1936). Further, to investigate host-parasite mediated horizontal transfer as a source of these elements, the genomes of three snail hosts corresponding to each of the major schistosomes, Biomphalaria glabrata, Oncomelania hupensis, and Bulinus truncatus, were examined in the same Southern blot as the six flatworms. Homo sapiens genomic DNA was also included in the analysis, since humans are the mammalian definitive host to all three major schistosome species.

\section{MATERIALS AND METHODS}

Source of DNA - Sources of genomic DNA used in the study were as follows. S. mansoni: cercariae (Puerto Rican strain), S. japonicum: adult worms (Chinese strain, Anhui Province), S. haematobium: adult worms (Egyptian strain), $O$. hupensis, B. truncatus, and B. glabrata: adult snails, $H$. sapiens: buffy coat isolated from non-schistosome infected human whole blood (provided by Fran Krogstad, Tulane University), F. hepatica: adult worms from Okeechobee, FL, US (provided by Dr Ray Kaplan, University of Georgia, Athens, GA, US) and Sydney, Australia (provided by Dr John Dalton, University of Technology, Sydney, Australia), F. magna: adult worms isolated from wild deer, central Florida, US (provided by Dr Ray Kaplan), and $D$. dorotocephala: adult worms (Carolina Biological Supply Co., Burlington, NC, US).

Extraction of genomic DNA - Samples preserved in ethanol were vacuum dried to remove the ethanol before extraction of genomic DNA. Genomic DNAs were extracted from the samples using the AquaPure Genomic DNA Purification system (Bio-Rad). In brief, tissue was lysed overnight at $55^{\circ} \mathrm{C}$ in a solution containing Proteinase $\mathrm{K}$ and, then treated with RNAse for $30 \mathrm{~min}$ at $37^{\circ} \mathrm{C}$ followed by 45 $\min$ at $65^{\circ} \mathrm{C}$. For whole blood, serum and most red blood cells were removed by centrifugation and isolation of the buffy coat. The buffy coat was then also subjected to treatment with a red blood cell lysis solution (Bio-Rad). Following RNAse treatment, samples were cooled, after which protein was removed by precipitation with salt. The supernatant was removed and DNA was precipitated with isopropanol, washed with ethanol, hydrated in sterile distilled water, and its concentration and purity determined by spectrophotmetric analysis at $\mathrm{OD}_{260}$ and $\mathrm{OD}_{280}$.

Gene probes, labeling of gene probes with ${ }^{32} P-d C T P$, Southern hybridization - The Boudicca-specific probe was produced by PCR amplification using the Le Paslier et al. (2000) bacterial artificial chromosome BAC 53-J-5 as a template and 5'-AACTGCAGATGCACGGAATCA CGGACT (forward) and 5'-GCTCTAGACTAAGATT CAGTCGGCAGATGC (reverse) primers, with restriction sites for $P s t \mathrm{I}$ and $\mathrm{Xba}$ I added to facilitate cloning into plasmid vectors. The probe, targeting part of the gag gene of Boudicca, was $385 \mathrm{bp}$ in length, spanning residues 694-1078 of the 5858 nucleotides of the 53-J-5 copy of Boudicca (AY662653). Identity was confirmed by sequencing. The Sinbad probe was obtained by PCR amplification of a fragment of the LePaslier et al. (2000) bacterial artificial chromosome BAC $30-\mathrm{H}-16$ using the primers 5'-CGCGGATCCAAGAGAAAAACCTTGATAGAC and 5'-CCGGAATTCCTGTCGAAGATAAAAGAGC. This fragment was cloned into pBluescript and its identity was confirmed by sequencing (Accession AY871176). The probe spans residues 2457 to 2823 of the BAC $33-\mathrm{N}-3$ copy of the Sinbad retrotransposon, the region of pol between the conserved protease and reverse transcriptase domains (Copeland et al. 2005a). The pSM389 probe is a $3.1 \mathrm{~kb}$ fragment encompassing the $3^{\prime}$ end of the intergenic spacer region and the $5^{\prime}$ end of the $18 \mathrm{~S}$ exon of the ribosomal RNA gene of S. mansoni (Vieira et al. 1991). This probe has been shown previously to hybridize not only with S. mansoni genomic DNA, but also with several species of Biomphalaria (Knight et al. 1991). This schistosome ribosomal gene probe was employed here alongside the retrotransposon based probes in order to verify the integrity and quantity of the genomic DNAs in the different lanes of the Southern blots (Fig. 1).

S. mansoni, S. japonicum, S. haematobium, O. hupensis, B. truncatus, B. glabrata, $H$. sapiens, $F$. hepatica, $F$. magna, and $D$. dorotocephala gDNA $(\sim 10 \mu \mathrm{g} /$ lane $)$ were digested with Hind III. Digested gDNA, along with 100 ng each of the probes described above, was size fractionated by electrophoresis through a $0.8 \%$ agarose gel, transferred to a nylon membrane (Zeta-Probe GT, Bio-Rad) by capillary action (Southern 1975), and UV-light cross-linked to the membrane.

The probes described above were labeled with ${ }^{32} \mathrm{P}$ radiolabeled dCTP using the RadPrime DNA Labeling System (Invitrogen). Five hundred nanograms of probe DNA were labeled according to the manufacturer's instructions. After pre-hybridization at $65^{\circ} \mathrm{C}$ in $0.25 \mathrm{M}$ $\mathrm{Na}_{2} \mathrm{HPO}_{4}, 7 \%$ SDS, 1 mM EDTA for 15 min, the radiolabeled probe was added to $20 \mathrm{ml}$ of fresh $0.25 \mathrm{M} \mathrm{Na}_{2} \mathrm{HPO}_{4}$, $7 \%$ SDS, $1 \mathrm{mM}$ EDTA and membranes were allowed to hybridize at $65^{\circ} \mathrm{C}$ in this solution overnight for high strin- 
gency hybridizations and $42^{\circ} \mathrm{C}$ overnight for low stringency hybridizations. The membranes were then washed twice in $40 \mathrm{mM} \mathrm{Na}_{2} \mathrm{HPO}_{4}, 5 \%$ SDS, $1 \mathrm{mM}$ EDTA for $1 \mathrm{~h}$ at $65^{\circ} \mathrm{C}$ (high stringency hybridizations) or $42^{\circ} \mathrm{C}$ (low stringency hybridizations) followed by two washes in $40 \mathrm{mM}$ $\mathrm{Na}_{2} \mathrm{HPO}_{4}, 1 \%$ SDS, $1 \mathrm{mM}$ EDTA, for $1 \mathrm{~h}$ each $\left(65^{\circ} \mathrm{C}\right.$ for high stringency hybridizations and $42^{\circ} \mathrm{C}$ for low stringency hybridizations). The membranes were then rinsed briefly in $2 \times \mathrm{SSC}$ and placed in clear plastic sheet protectors attached to BioMax amplification screens (Kodak). The signals from the ${ }^{32} \mathrm{P}$-labeled gene probes were detected using x-ray film (Fuji).

Separate blots were used for the Sinbad and Boudicca hybridizations, and both these blots were subsequently stripped and probed with pSM389 as a positive control. The blots were stripped as follows. After briefly rinsing the membranes in $2 \times \mathrm{SSC}$, the membranes were washed four times by immersion in $0.1 \% \mathrm{SDS}, 0.1 \times \mathrm{SSC}$ at $100^{\circ} \mathrm{C}$ followed by washing $\left(37^{\circ} \mathrm{C}\right.$, rocking) for $15 \mathrm{~min}$ as the solution cooled. No radioactivity was detected from the membranes by Geiger counter after these four washes.

EST searches - EST searches for Boudicca and Sinbad elements in the $S$. japonicum transcriptome were carried out using the complete sequence of each retrotransposon (accession number AY662653 for Boudicca and AY506538 for Sinbad). The search parameters were set to search the EST database of S. japonicum only, with "Schistosoma japonicum[organism]" entered into the "Limit by entrez entry" box of the "options for advanced blasting" section.

\section{RESULTS}

Sinbad-like elements present in S. haematobium Standard (high stringency) Southern hybridization was used to search for Sinbad-like retrotransposons in the genomes of S. japonicum and S. haematobium, in the genomes of helminths phylogenetically related to these schistosomes, and in the genomes of host organisms parasitized by schistosomes (human and snails). The pattern of restriction fragments of $S$. mansoni genomic DNA was consistent with that of previous experiments. The Sinbad element was found only in S. mansoni and S. haematobium. Its presence was not evident in the genomes of any of the other flatworms, or in the snail hosts, or in H. sapiens (Fig. 2, the signal seen in the Dugesia lane is extended radiation from the positive control lane's signal.) The $S$. haematobium signal showed a clear clustering in the $~ 11$ $14 \mathrm{~kb}$ range, whereas the signal in $S$. mansoni was intense to large fragments of $>15 \mathrm{~kb}$ in size (Fig. 2), a reflection of the general Hind III digestion pattern for these genomes (Fig. 1B).

In the $1-4 \mathrm{~kb}$ range, the patterns shown by the two species were similar (Fig. 2). Hind III is not expected to cut within Sinbad, as indicated by the fully sequenced Sinbad copy found in Le Paslier et al. (2000) BAC library clone 33-N-3 (Copeland et al. 2005a) but has been found to cut once in a Sinbad fragment found at the end of the BAC 30-H-16 clone of Le Paslier et al. (2000).

Boudicca-like elements present in S. haematobium These same ten genomes were examined for the presence

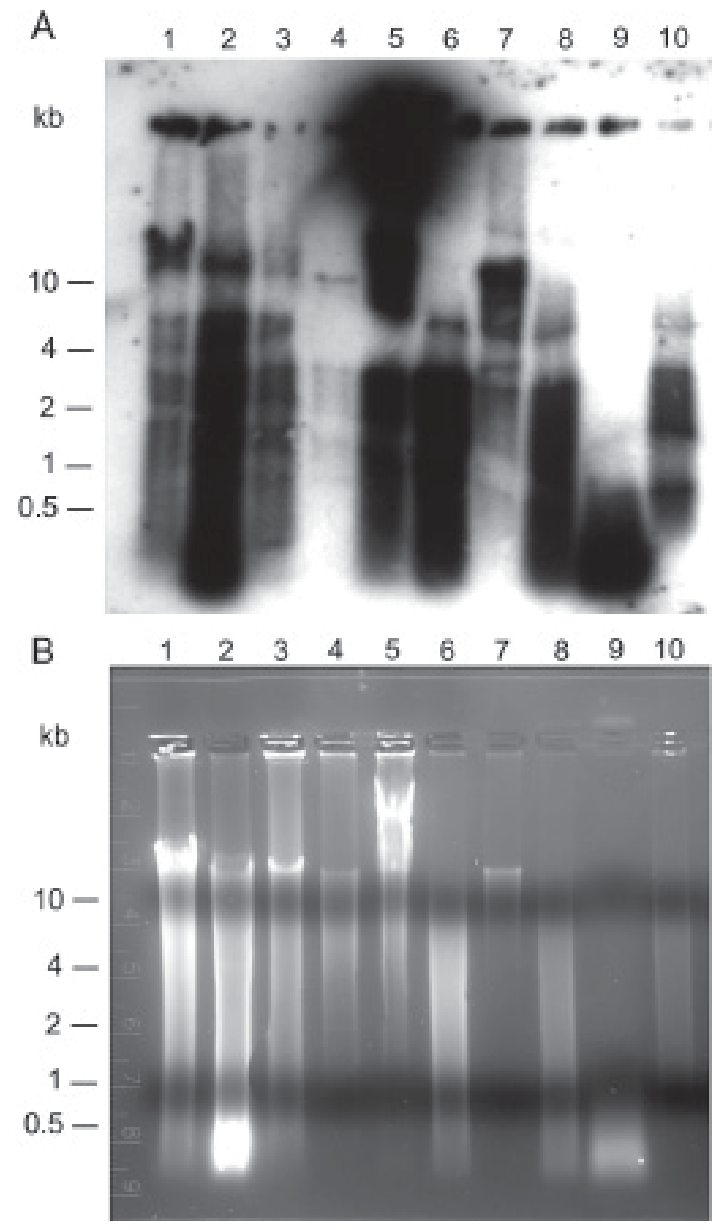

Fig. 1A: Southern hybridization analysis of Hind III digested genomic DNA from 10 genomes, probed with a radiolabeled Schistosoma mansoni ribosomal gene specific probe and exposed to X-ray film for 14 days. Size scale is shown to the left, in kilobases. Lanes - 1: Oncomelania hupensis; 2: Bulinus truncatus; 3: Biomphalaria glabrata; 4: Homo sapiens; 5: S. mansoni; 6: S. japonicum; 7: S. haematobium; 8: Fasciola hepatica; 9: Fascioloides magna; 10: Dugesia dorotocephala; B: ethidium stained gel of Hind III digested genomic DNA from 10 genomes used for the blot depicted in (A). Size scale is shown to the left, in kilobases. Lanes - 1: $O$. hupensis; 2: B. truncatus; 3: B. glabrata; 4: H. sapiens; 5: S. mansoni; 6: S. japonicum; 7: S. haematobium; 8: F. hepatica; 9: F. magna; 10: D. dorotocephala.

of a Boudicca-like retrotransposon, also using standard Southern hybridization. As with Sinbad, only S. mansoni and $S$. haematobium were found to be positive for Boudicca (Fig. 3). Both S. mansoni and S. haematobium exhibited areas of strong signal at $\sim 8-11 \mathrm{~kb}$ and $\sim 3 \mathrm{~kb}$ (Fig. 3). On the basis of the fully sequenced copy of Boudicca (from BAC 53-J-5), Hind III is expected to cut twice within the retrotransposon, at positions 4470 and 4793 of the 5858 bp element, yielding a $\sim 300$ bp product. The $S$. haematobium signal showed a distinct band at $\sim 500$ bp (Fig. 3), and S. mansoni gave strong signals for smaller fragments. As this copy of Boudicca exhibits several mutations (Copeland et al. 2003), the Hind III restriction map may differ in other Boudicca copies. 


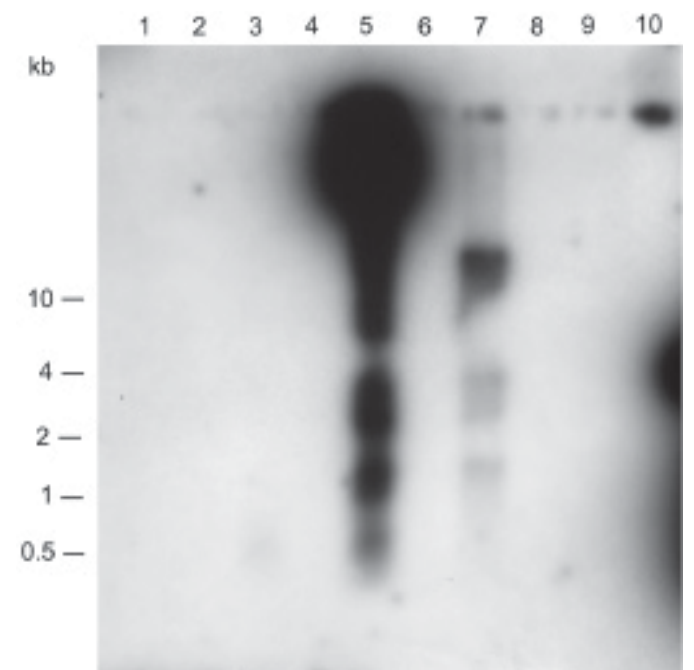

Fig. 2: Southern hybridization analysis of Hind III digested genomic DNA from 10 genomes, probed with a radiolabeled Sinbad specific probe and exposed to X-ray film for 14 days. Size scale is shown to the left, in kilobases. Lanes - 1: Oncomelania hupensis, 2: Bulinus truncatus; 3: Biomphalaria glabrata; 4: Homo sapiens; 5: Schistosoma mansoni; 6: S. japonicum; 7: S. haematobium; 8: Fasciola hepatica; 9: Fascioloides magna; 10: Dugesia dorotocephala.

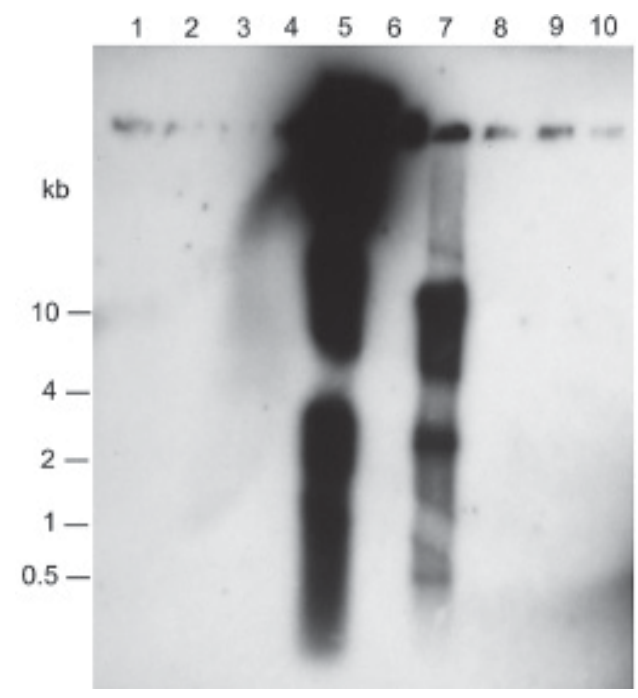

Fig. 3: Southern hybridization analysis of Hind III digested genomic DNA from 10 genomes, probed with a radiolabeled Boudicca specific probe and exposed to X-ray film for 10 days. Size scale is shown to the left, in kilobases. Lanes - 1: Oncomelania hupensis; 2: Bulinus truncatus; 3: Biomphalaria glabrata; 4: Homo sapiens; 5: S. mansoni; 6: S. japonicum; 7: Schistosoma haematobium; 8: Fasciola hepatica; 9: Fascioloides magna; 10: Dugesia dorotocephala.

S. mansoni ribosomal gene probe - As a positive control, a probe based on the $18 \mathrm{~S}$ ribosomal subunit and preceding intergenic spacer of $S$. mansoni (Ali et al. 1991) was used in a final standard Southern hybridization. This probe, known as pSM389, has been shown previously to hybridize not only with $S$. mansoni genomic DNA, but also with several species of Biomphalaria (Knight et al. 1991). We hypothesized that the conserved nature of ri- bosomal genes would allow this probe to be used as a positive control for all of the genomes examined in this study. The probe did indeed hybridize to all species, with strong signals for the trematodes and snails and a weak but discernable signal for the human DNA (Fig. 1a). From these results, it can be concluded that the gDNA of these ten species transferred successfully to the blot, and that negative results for species such as $S$. japonicum do not reflect a lack of cross-linked DNA.

Low stringency hybridization suggests Boudicca-like elements in B. truncatus - In addition to the high stringency Southern hybridizations described above (overnight hybridization at $65^{\circ} \mathrm{C}$ ), low stringency hybridizations (overnight hybridization at $42^{\circ} \mathrm{C}$ ) were performed on the same blots with both the Boudicca and Sinbad probes. The Sinbad low stringency results resembled the high stringency results shown in Fig. 2, with only the $S$. mansoni and S. haematobium lanes exhibiting a positive signal (not shown). The Boudicca blot, however, exhibited a pattern significantly different from that of the high stringency hybridization (Fig. 4). Though the S. mansoni and S. haematobium lanes exhibited the strongest signals, the next strongest signal, unexpectedly, was found in the B. truncatus lane. The signal for Bulinus (the snail host of $S$. haematobium) was stronger than that of $S$. japonicum, almost as strong as that of $S$. haematobium, and significantly stronger than any of the signals from the other genomes examined.

In all three blots, the strong signal toward the top of the blot seen in $S$. mansoni but not in S. haematobium reflects the general pattern of Hind III digested S. mansoni genomic DNA, with a large proportion of relatively undigested DNA (large fragments $>15 \mathrm{~kb}$ in size) compared to other genomes examined (Fig. 1B). Likewise, the strong signal around $11-14 \mathrm{~kb}$ in $S$. haematobium reflected the general restriction pattern for that schistosome.

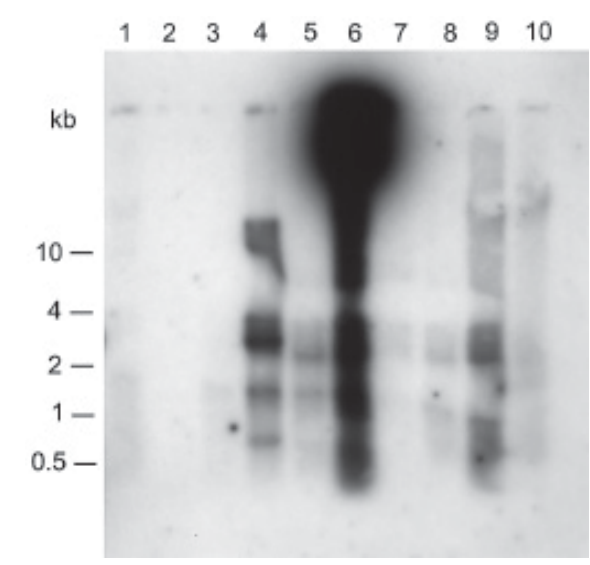

Fig. 4: low stringency Southern hybridization analysis of Hind III digested genomic DNA from 10 genomes, probed with a radiolabeled Boudicca specific probe and exposed to X-ray film for 30 days. Size scale is shown to the left, in kilobases. Lanes - 1: Dugesia dorotocephala; 2: Fascioloides magna; 3: Fasciola hepatica; 4: Schistosoma haematobium; 5: S. japonicum; 6: S. mansoni; 7: Homo sapiens; 8: Biomphalaria glabrata; 9: Bulinus truncatus; 10: Oncomelania hupensis. 


\section{DISCUSSION}

Of the three major species of schistosomes, S. haematobium is the least understood in terms of genome sequence. Until now, no LTR retrotransposons have been found in S. haematobium, though the presence of the (SINE-like) Sma elements (Ferbeyre et al. 1998) indicates that non-LTR retrotransposons are probably present. This study represents the first evidence of the presence of LTR retrotransposons in the genome of S. haematobium. Given the general paucity of genomic data for $S$. haematobium, and the fact that Boudicca and Sinbad elements make up over $4 \%$ of the S. mansoni genome (Copeland et al. 2005b), these findings significantly advance the exploration of the genome of this important human parasite. In addition, transposable elements are of interest for their possible utility in applications related to both sequencing and manipulation of genomes, including transgenesis and gene knockouts, generation of cell culture lines, generation of mutant libraries, and increased accuracy in whole genome shotgun sequencing (Izsvak et al. 1997, Handler 2001, Hirochika 2001, Tammi et al. 2003). Knowledge of these elements from S. haematobium will aid in the process of molecular genetic characterization of the $S$. haematobium genome.

$S$. haematobium is considered to be much more closely related to the other African species, S. mansoni, than to $S$. japonicum, an Asian species. In addition, evidence based on ribosomal RNA gene sequence, gene order of the mitochondrial genomes, and C-banding patterns of nuclear genome karyotypes suggests that $S$. japonicum is ancestral to both $S$. mansoni and S. haematobium (Snyder \& Loker 2000, Le et al. 2000, Hirai et al. 2000). The presence of Sinbad and Boudicca in S. haematobium and $S$. mansoni, but not $S$. japonicum, is consistent with this phylogeny, and could have been brought about by the acquisition of the elements after the split from the $S$. japonicum lineage but before the divergence of $S$. mansoni and S. haematobium. Alternatively, ancestral elements of Boudicca and Sinbad could have been present in a basal ancestral schistosome, and subsequently diverged into different elements or were lost from the $S$. japonicum genome after the split between the $S$. japonicum and $S$. mansoni/S. haematobium lineage.

If these retrotransposons had ancient origins in the ancestors of $S$. mansoni, it would be expected that the genomes of related species would also include at least vestigial forms of these elements. However, neither the moderately related turbellarian and trematode genomes, nor the closely related $S$. japonicum genome appeared to contain these elements. Thus far, no Boudicca elements have been identified in S. japonicum, and the only Paolike element from S. japonicum, Tiao (Fan \& Brindley 1998) has not been fully characterized. A BLAST search of the $S$. japonicum EST database with the complete sequence of the Boudicca retrotransposon (AY662653) did reveal a match to a reverse transcriptase (BU723196) with moderate similarity (bit score $=97$ ) to that of Boudicca, indicating that $S$. japonicum may encode, and transcribe, Gypsygroup LTR retrotransposons from the CsRn1/Kabuki clade. This clade, of which Boudicca is a member, con- sists of elements so far found only in the genomes of Bombyx mori, Chlonorchis sinensis, Paragonimus westermani, and S. mansoni, and is defined by a unique cyshis box structure (Copeland et al. 2005b). A corresponding BLAST search using the complete sequence of the Sinbad retrotransposon (AY506538) failed to yield any significant matches. More detailed characterization of Tiao and any $S$. japonicum elements from the $C s R n 1 / K a$ buki clade, would help to elucidate this phylogeny.

The results of this study indicate either a relatively recent origin (after the split between the $S$. japonicum and S. mansoni/S. haematobium lines) for the Boudicca and Sinbad retrotransposons via horizontal transmission, the loss of these elements in the S. japonicum lineage, or a high degree of divergence of ancestral retrotransposons of Boudicca and Sinbad after the split between the $S$. japonicum and $S$. mansoni/S. haematobium lineage. Previously, elements closely related to Boudicca have been found only in platyhelminths and insects (Bae et al. in press, Copeland et al. 2005b), whereas elements known to be closely related to Sinbad have been limited to schistosomes and fish (Copeland et al. 2005a). The presence of a robust positive signal for Sinbad in S. haematobium but absence in $S$. japonicum, the other platyhelminths, and the host genomes, even under low stringency conditions, supports the possibility of a horizontal transfer event from some other genome after the divergence of $S$. japonicum from the S. mansoni/S. haematobium line. The finding of a Sinbad-like element in the zebrafish Danio rerio, combined with the presence of other Pao-like elements in the genomes of aquatic, but not terrestrial, vertebrates (Copeland et al. 2005a), suggests a possible origin for Sinbad in fish.

The robust positive signal for Boudicca in Bulinus under low stringency conditions raises the possibility of a horizontal transfer event of an ancestral Boudicca-like element from an ancestral snail to an ancestral schistosome. Especially intriguing is the strength of the signal; the Bulinus signal was not only stronger than that of the platyhelminth genomes but was also stronger than that of the host genome of S. mansoni, Biomphalaria. The evolutionary path suggested by this pattern is a jump from an ancestral Bulinus snail genome to an ancestral $S$. haematobium/S. mansoni genome, which in turn points to Bulinus or an ancestor of Bulinus as the original host of this ancestral schistosome. One caveat, considering the low stringency conditions of the experiment, is that it is plausible for an element only moderately related to Boudicca to generate a strong signal if it were very high in copy number. The fact that the positive signal in Bulinus only emerged under low stringency conditions therefore necessitates further inquiry as to the identity of any Boudicca-like elements in this genome, and invites further studies of Boudicca-like elements in other snail genomes.

\section{ACKNOWLEDGEMENTS}

To Dr Ray Kaplan and Dr John Dalton for the donation of Fascioloides magna and Fasciola hepatica parasites, and to Fran Krogstad for supplying human blood. 


\section{REFERENCES}

Ali PO, Simpson AJ, Allen R, Waters AP, Humphries CJ, Johnston DA, Rollinson D 1991. Sequence of a small subunit rRNA gene of Schistosoma mansoni and its use in phylogenetic analysis. Mol Biochem Parasitol 46: 201208.

Bae YA, Kong Y in press. Retrotransposons in the genomes of the digenean parasitic trematodes, Clonorchis sinensis and Paragonimus westermani. In PJ Brindley, Mobile Genetic Elements in Metazoan Parasites, Eurekah/Landes Bioscience, Georgetown, TX.

Brindley PJ 2005. The molecular biology of schistosomes. Trends Parasitol 21: 533-536.

Brindley PJ, Laha T, McManus DP, Loukas, A 2003. Mobile genetic elements colonizing the genomes of metazoan parasites. Trends Parasitol 19: 79-87.

Charlesworth B, Sniegowski P, Stephan W 1994. The evolutionary dynamics of repetitive DNA in eukaryotes. Nature 371: 215-220.

Copeland CS, Brindley PJ, Heyers O, Michael SF, Johnston DA, Williams DJ, Ivens A, Kalinna BH 2003. Boudicca, a retrovirus-like, LTR retrotransposon from the genome of the human blood fluke, Schistosoma mansoni. J Virol 77: 6153-6166.

Copeland CS, Heyers O, Kalinna BH, Bachmair A, Stadler PF, Hofacker IL, Brindley PJ 2004. Structural and evolutionary analysis of the transcribed sequence of Boudicca, a Schistosoma mansoni retrotransposon. Gene 329: 103-114.

Copeland CS, Laha T, Brindley PJ 2005b. Schistosome long yerminal repeat retrotransposons. In PJ Brindley, Mobile Genetic Elements in Metazoan Parasites, Eurekah/Landes Bioscience, Georgetown, TX.

Copeland CS, Mann VH, Morales ME, Kalinna BH, Brindley PJ 2005a. The Sinbad retrotransposon from the genome of the human blood fluke, Schistosoma mansoni, and the distribution of related Pao-like elements. BMC Evol Biol 5: 20.

DeMarco R, Kowaltowski AT, Machado AA, Soares MB, Gargioni C, Kawano T, Rodrigues V, Madeira AM, Wilson RA, Menck CF, Setubal JC, Dias-Neto E, Leite LC, Verjovski-Almeida S 2004. Saci-1, -2, and -3 and Perere, four novel retrotransposons with high transcriptional activities from the human parasite Schistosoma mansoni. J Virol 78: 2967-2978.

Drew A, Brindley PJ 1995. Female-specific sequences isolated from Schistosoma mansoni by representational difference analysis. Mol Biochem Parasitol 71: 173-181.

El-Sayed NM, Bartholomeu D, Ivens A, Johnston DA, LoVerde PT 2004. Advances in schistosome genomics. Trends Parasitol 20: 154-157.

Fan J, Brindley PJ 1998. Retrotransposable elements in the Schistosoma japonicum genome. In SK Isao Tada, Moriyasu Tsuji (eds), Ninth International Congress of Parasitology, Monduzzi Editore, Makuhari Messe, Chiba, Japan, p. 821825 .

Ferbeyre G, Smith JM, Cedergren R 1998. Schistosome satellite DNA encodes active hammerhead ribozymes. Mol Cell Biol 18: 3880-3888.

Feschotte C 2004. Merlin, a new superfamily of DNA trans- posons identified in diverse animal genomes and related to bacterial IS1016 insertion sequences. Mol Biol Evol 21: 1769-1780.

Foulk BW, Pappas G, Hirai Y, Hirai H, Williams DL 2002. Adenylosuccinate lyase of Schistosoma mansoni: gene structure, mRNA expression, and analysis of the predicted peptide structure of a potential chemotherapeutic target. Int $J$ Parasitol 32: 1487-1495.

Garfinkel DJ, Hedge AM, Youngren SD, Copeland TD 1991. Proteolytic processing of pol-TYB proteins from the yeast retrotransposon Ty1. J Virol 65: 4573-4581.

Handler AM 2001. A current perspective on insect gene transformation. Insect Biochem Mol Biol 31: 111-128.

Havecker ER, Gao X, Voytas DF 2004. The diversity of LTR retrotransposons. Genome Biol 5: 225.

Hillyer GV 1974. Buoyant density and thermal denaturation profiles of schistosome DNA. J Parasitol 60: 725-727.

Hirai H, Taguchi T, Saitoh Y, Kawanaka M, Sugiyama H, Habe S, Okamoto M, Hirata M, Shimada M, Tiu WU, Lai K, Upatham ES, Agatsuma T 2000. Chromosomal differentiation of the Schistosoma japonicum complex. Int J Parasitol 30: 441-452.

Hirochika H 2001. Contribution of the Tos 17 retrotransposon to rice functional genomics. Curr Opin Plant Biol 4: 118122.

Hu W, Yan Q, Shen D-K, Liu F, Xu X-R, Zhu Z-D, Wu X-W, Zhang X, Wang J-J, Xu X, Wang Z, Huang J, Wang S-Y, Wang Z-Q, Brindley PJ, McManus DP, Xue C-L, Feng F, Chen Z, Han Z-G 2003. Evolutionary and biomedical implications of a Schistosoma japonicum complementary DNA resource. Nat Genet 35: 139-147.

Izsvak Z, Ivics Z, Hackett PB 1997. Repetitive elements and their genetic applications in zebrafish. Biochem Cell Biol 75: $507-523$.

Kazazian HH, Jr 2004. Mobile elements: drivers of genome evolution. Science 303: 1626-1632.

Kidwell MG, Lisch D 1997. Transposable elements as sources of variation in animals and plants. Proc Natl Acad Sci USA 94: 7704-7711.

Knight M, Brindley PJ, Richards CS, Lewis FA 1991. Schistosoma mansoni: use of a cloned ribosomal RNA gene probe to detect restriction fragment length polymorphisms in the intermediate host Biomphalaria glabrata. Exp Parasitol 73: 285-294.

Laha T, Brindley PJ, Smout MJ, Verity CK, McManus DP, Loukas A 2002a. Reverse transcriptase activity and untranslated region sharing of a new RTE-like, non-long terminal repeat retrotransposon from the human blood fluke, Schistosoma japonicum. Int J Parasitol 32: 1163-1174.

Laha T, Brindley PJ, Verity CK, McManus DP, Loukas A 2002b. pido, a non-long terminal repeat retrotransposon of the chicken repeat 1 family from the genome of the Oriental blood fluke, Schistosoma japonicum. Gene 284: 149-159.

Laha T, Loukas A, Smyth DJ, Copeland CS, Brindley PJ 2004. The fugitive LTR retrotransposon from the genome of the human blood fluke, Schistosoma mansoni. Int J Parasitol 34: 1365-1375. 
Laha T, Loukas A, Verity CK, McManus DP, Brindley PJ 2001. Gulliver, a long terminal repeat retrotransposon from the genome of the oriental blood fluke Schistosoma japonicum. Gene 264: 59-68.

Laha T, McManus DP, Loukas A, Brindley PJ 2000. Sjalpha elements, short interspersed element-like retroposons bearing a hammerhead ribozyme motif from the genome of the oriental blood fluke Schistosoma japonicum. Biochim Biophys Acta 1492: 477-482.

Le Paslier MC, Pierce RJ, Merlin F, Hirai H, Wu W, Williams DL, Johnston D, LoVerde PT, Le Paslier D 2000. Construction and characterization of a Schistosoma mansoni bacterial artificial chromosome library. Genomics 65: 87-94.

Le TH, Blair D, McManus DP 2000. Mitochondrial DNA sequences of human schistosomes: the current status. Int $J$ Parasitol 30: 283-290.

Mostafa MH, Sheweita SA, O'Connor PJ 1999. Relationship between Schistosomiasis and bladder cancer. Clin Microbiol Rev 12: 97-111.

Petrov D, Sangster TA, Johnston JS, Hartl DL, Shaw KL 2000. Evidence for DNA loss as a determinant of genome size. Science 287: 1060-1062.

Rollinson D, Kaukas A, Johnston DA, Simpson AJ, Tanaka M 1997. Some molecular insights into schistosome evolution. Int J Parasitol 27: 11-28.

Snyder SD, Loker ES 2000. Evolutionary relationships among the Schistosomatidae (Platyhelminthes:Digenea) and an Asian origin for Schistosoma. J Parasitol 86: 283-288.

Southern, EM 1975. Detection of specific sequences among DNA fragments separated by gel electrophoresis. $\mathrm{J} \mathrm{Mol}$
Biol 98: 503-517.

Spotila L, Hirai H, Rekosh DM, Lo Verde PT 1989. A retroposon-like short repetitive DNA element in the genome of the human blood fluke, Schistosoma mansoni. Chromosoma 97: 421-428.

Swales WE 1936. Further studies on Fascioloides magna (Bassi, 1875) Ward, 1917, as a parasite of ruminants. Can J Res 14: 83-95.

Tammi MT, Arner E, Kindlund E, Andersson B 2003. Correcting errors in shotgun sequences. Nucleic Acids Res 31: 4663-4672.

Verjovski-Almeida S, DeMarco R, Martins EA, Guimaraes PE, Ojopi EP, Paquola AC, Piazza JP, Nishiyama MY, Jr., Kitajima JP, Adamson RE, Ashton PD, Bonaldo MF, Coulson PS, Dillon GP, Farias LP, Gregorio SP, Ho PL, Leite RA, Malaquias LC, Marques RC, Miyasato PA, Nascimento AL, Ohlweiler FP, Reis EM, Ribeiro MA, Sa RG, Stukart GC, Soares MB, Gargioni C, Kawano T, Rodrigues V, Madeira AM, Wilson RA, Menck CF, Setubal JC, Leite LC, Dias-Neto E 2003. Transcriptome analysis of the acoelomate human parasite Schistosoma mansoni. Nat Genet 35: 148-157.

Vieira LQ, Correa-Oliveira R, Katz N, de Souza CP, Carvalho OS, Araujo N, Sher A, Brindley PJ 1991. Genomic variability in field populations of Schistosoma mansoni in Brazil as detected with a ribosomal gene probe. Am J Trop Med Hyg 44: 69-78.

Xiong Y, Burke WD, Eickbush TH 1993. Pao, a highly divergent retrotransposable element from Bombyx mori containing long terminal repeats with tandem copies of the putative R region. Nucleic Acids Res 21: 2117-2123. 